\title{
An FEM Analysis with Consideration of Random Void Defects for Predicting the Mechanical Properties of 3D Braided Composites
}

\author{
Kun Xu and Xiaomei Qian \\ School of Aeronautics and Astronautics, University of Electronic Science and Technology of China, Chengdu 611731, China \\ Correspondence should be addressed to Kun Xu; xukun@uestc.edu.cn
}

Received 9 May 2013; Revised 4 December 2013; Accepted 4 December 2013; Published 19 February 2014

Academic Editor: Rui Huang

Copyright @ $2014 \mathrm{~K}$. Xu and X. Qian. This is an open access article distributed under the Creative Commons Attribution License, which permits unrestricted use, distribution, and reproduction in any medium, provided the original work is properly cited.

Random void defects have detrimental effect on the mechanical properties of 3D braided composites. A 3D FEM based on a periodic representative unit cell is developed to appraise the mechanical properties of 3D 4-directional braided composites containing the defects. Two basic types of void defects, such as the dry patches in the reinforced yarns and the voids in the resin matrix pocket, have been taken into account. A simple method for generating the random void defect elements in FEM is presented. The FE software ABAQUS is adopted to study the elastic properties. The predicted effective elastic properties are in good agreement with the available experimental data, demonstrating the applicability of the mesomechanical FEM. By considering the random distribution of void defects, the probability statistics analysis of mechanical properties was conducted. In addition, the effect of the void volume fraction on the elastic properties was discussed in detail, and some useful conclusions were drawn herein.

\section{Introduction}

Textile fabrics composites have received great attention in the aeronautics and astronautics industries because of their excellent mechanical performances, such as better out-ofplane stiffness, strength, and high impact resistance. To make these composites widely applied in structure design as soon as possible, many scholars have developed lots of models for studying the microstructure and mechanical properties of textile fabrics composites.

In the past, Whitcomb et al. [1-3] established the localglobal finite element method to analyze the progressive failure for textile composites. Tang and Whitcomb $[4,5]$ proposed the general techniques for failure analysis for textile composites by considering their materials features such as periodicity, symmetries, and heterogeneity. Lomov et al. [6] formulated a model for woven fabric behavior in bending based on a representation of fabric deformation as a coordinate transformation. Verpoest and Lomov [7] developed the software package WiseTex to implement a generalized description of internal structure of textile reinforcements on the unit cell level and it is integrated with mechanical models for several types of textile composites. In recent years, Yang et al. [8] analyzed the failure in the junction region of Tstiffeners regarding 3D-braided and 2D tape laminate stiffeners. Yang et al. $[9,10]$ studied the spatially averaged local strains in textile composites by the binary model. Meanwhile, they predicted the failure behavior in textile composites by the model with gauge-averaging [11]. To reduce the number of costly composites tests, Cox and Yang [12] studied the virtual tests for structural composites and proposed the refinement of cohesive models of fracture and the formulation of hybrid stress-strain and traction-displacement models that combine continuum (spatially averaged) and discrete damage representations in a single calculation. Karkkainen et al. [13, 14] presented a direct micromechanics method for failure analysis and strength prediction of plain weave textile composites and investigated the stiffness and the strength of $3 \mathrm{D}$ orthogonal woven S2-glass composite by using finite element micromechanics [15]. Quek et al. [16] studied the effective elastic stiffness and strength of a carbon 2D triaxially braided composite by using the finite element method in conjunction 
with micromechanics. Pineda et al. [17] presented a model to model progressive microdamage in the matrix phase by the thermodynamically based Schapery theory.

As an important type of textile fabrics composites, 3D braided composites have been studied by many scholars and many models have been developed to analyze the mechanical performance of 3D braided composites. For example, Yang et al. [18] early studied the effective elastic properties of 3D braided composites by the "fiber inclination model" based on the modified laminate theory. Y. -Q. Wang and A. S. D. Wang [19] and X. K. Sun and C. J. Sun [20] adopted a mixed volume averaging technique to predict the mechanical properties of $3 \mathrm{D}$ braided composites, respectively. Chen et al. [21] analyzed the mechanical properties of $3 \mathrm{D}$ braided composites by the finite multiphase element method. K. Xu and X. W. Xu [22] predicted the mechanical properties of 3D five-directional braided composites by finite element method. Lu et al. [23] studied the tensile properties of three-dimensional full fivedirectional braided composites based on representative unit cell by using finite element methods.

Although a number of analytical and computational models have contributed to an enhanced understanding of the elastic properties of 3D braided composites, most of the models have generally assumed that braided composites have no void defects and do not consider the effect of random void defects on their elastic properties. However, since 3D braided composites are manufactured with the continuous fiber preforms by RTM forming process, it is almost impossible to completely avoid the existence of void defects in these composites. Now, there are few literatures focusing on the effect of the void defects on the elastic properties of $3 \mathrm{D}$ braided composites. Liang et al. [24] adopted the Eshelby and MoriTanaka theories combined with stiffness averaging method to predict the elastic constants of $3 \mathrm{D}$ braided composites containing the specific matrix microcracks. Zeng et al. [25] proposed a simplified model to predict effective Young's modulus and Poisson's ratio of 3D braided composites with transverse and longitudinal cracks by using the homogenization theorem. Lu et al. [26] developed a model to simulate the unidirectional tension behavior of plain weave composites with defects. As a whole, these early models aiming at cracks consider the architectures of 3D braided composites to be simplified, which made it difficult to obtain an accurate local stress distribution of unit cell model.

Recently, great efforts have been made to further investigate the microstructure model of textile composites for improving the exactness of microstructure model by using high resolution experimental techniques. Bale et al. [27] performed a statistical analysis of the shape and positioning of the fiber tows in the 3D woven architecture on the basis of 3D images of two ceramic-matrix textile composites captured by X-ray micron-resolution computed tomography. Blacklock et al. [28] presented a Monte Carlo algorithm defined for generating replicas of textile composite specimens by using the computed tomography, which help us identify the geometry characteristics of textile composites.

In the paper, the main objective of the work is to develop a 3D FEM based on a unit cell for predicting the elastic properties of 3D four-directional braided composites containing the random void defects. Firstly, the void defect characteristics of 3D braided composites by RTM process are analyzed. Then, based on a microstructure unit cell model of 3D four-directional braided composites, an FEM considering the random distribution of typical void defects is proposed. The FE software ABAQUS is adopted to calculate the elastic properties. The predicted effective elastic properties are in good agreement with the available experimental data, demonstrating the applicability of the mesomechanical FEM. In the meantime, the effect of random distribution of void defect on the elastic properties of the composites with a constant void volume fraction is analyzed by establishing several different samples. Finally, in order to appraise the effect of the void defects on the elastic properties, the variations of the elastic properties with the void volume fraction are discussed in detail.

\section{Void Defect Characteristics of 3D Braided Composites}

Liquid composite molding (LCM) processes such as RTM and vacuum assisted resin transfer molding (VARTM) are widely used to manufacture the advanced textile composites with the continuous fiber reinforcements. In the process, a dry fiber preform is placed in the mold and a thermoset resin is injected into it to cover the empty spaces between fibers. Most experimental investigations indicated that voids and dry patches are the most common types of manufacturing process induced defects in the textile composite materials [29-31]. The presence of voids and dry patches, even at a very low volume fraction, may significantly degrade the material properties and have detrimental effect on the service performance. Therefore, resin infiltration into the preforms should be carefully controlled in order to minimize the residual voids content, which may produce the probable voids or dry patches of the composites.

Two basic flow directions for resin may exist in the RTM forming process, which are flow across or along fiber tows. In the case of flow across the fiber tows, lots of experimental evidence and calculated results have shown that filling of the fiber tows is delayed. If the resin is not forced to flow only across the fiber tows, naturally, flow will choose the easier direction with higher permeability, that is, along the fiber tows. Compared with flow across the tows, the permeability of flow along the fiber tows is much higher and capillary action is generally twice as strong as in flow across the tows. In this case, two situations can be found in flow along the fiber tows. For example, as given in Figure 1, with respect to the primary front in the intertow spaces, wicking flow front inside the fiber tow may be either advanced or delayed, which also directly has great effect on the location and shape of emerging voids. When the externally applied action is lower, wicking flow can become dominant and resin advances more rapidly inside the tows. In this case, the voids in the resin matrix pocket are formed as shown in Figure 1(a). Reversely, 


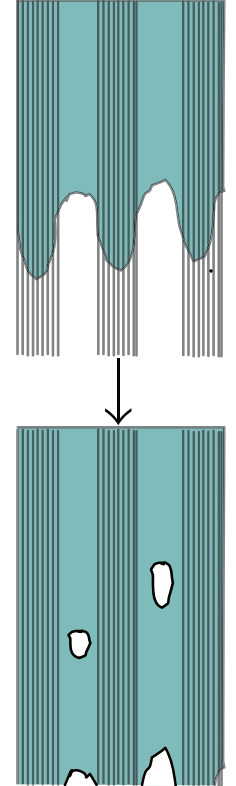

(a) Intertow void generation



(b) Intratow void generation
FIGURE 1: Illustration of inter- and intratow void generation.

the externally applied pressure or flow rate is relatively high or fast, viscous action is dominant, and wicking gradient is not so strong compared with the hydrodynamic pressure gradient. In this case, the intertow spaces with the higher permeability regions are filled first and the dry patches of tows are produced as shown in Figure 1(b). If an equilibrated situation occurs, no voids are to be generated because of the resin progressing more or less uniformly.

As above discussed briefly, the production of voids is controlled by the key manufacturing parameters such as externally applied pressure, resin viscosity, and curing temperature. Besides, the specific manufacturing process is an important control factor for void defects generation of 3D braided composites. Although the reasons for defect formation are very complicated, air or volatile gas in the mold is captured mechanically to finally form the residual voids of the composites. For 3D braided composites with the complex microstructure, the void defects are impossible to be expected to form somewhere either in yarns or in resin matrix pocket due to the complex reasons. Therefore, once the defects are produced, the resultant voids can be called the random defects and have the random distribution feature. While the voids existing in yarns are called the dry patch defects, the defects existing in resin matrix pocket are called the void defects.

For the defects of 3D braided composites, they generally have very complicated geometrical shapes. For example, Figure 2 gives the cross-sectional shape of the voids in resin matrix pocket of $3 \mathrm{D}$ braided composites by vacuum assisted RTM, which is the complex geometry polygon [29]. By using the image analysis software tool, the equivalent diameters of the typical voids can be measured. Formula (1) gives the expression for the equivalent diameters of void:

$$
D_{\mathrm{eq}}=2 \sqrt{\frac{A}{\pi}},
$$

where $D_{\mathrm{eq}}$ is the equivalent diameter and $A$ is the measured area of the void. Extensive experimental analysis has indicated that the type of RTM process will lead to producing different void size of 3D braided composites [29]. Based on the statistical analysis, the equivalent diameter of void size is generally less than $300 \mu \mathrm{m}$.

\section{Unit Cell Model}

3D four-directional rectangular braided composites reported herein are produced by the 4 -step $1 \times 1$ braiding procedure. According to the rectangular cross-sectional shape, the yarns carriers in the main part of the braiding machine bed are commonly denoted by $M \times N, M$ being the number of rows and $N$ being the number of columns, which can almost determine the cross-sectional dimensions of the rectangular braided composites with certain yarns. To ensure consistent and uniform fabric structure, suppose that the braiding procedure keeps relatively steady, at last in a specified length of braiding. As a result, a braiding pitch with the height of $h$ is obtained for 3D four-directional rectangular braided composites.

Because of the complexity of braided structures, many models based on the interior unit cell models in the mesoscale have been presented to analyze the mechanical properties of $3 \mathrm{D}$ braided composites in the macroscale. In the paper, an interior unit cell model is adopted to represent 3D fourdirectional braided composites with mesoscale periodic unit cells, as shown in Figure 3. The interior unit cell model is oriented in the same reference frame as the specimen cross-section, which is quite favorable for the analysis of the mechanical properties.

Figure 3 shows the topological relation of the main yarns in a parallelepiped unit cell with the width of $W_{i}$, the thickness of $T_{i}$, and the pitch height of $h . \alpha$ is the braiding angle between the grain formed by the adjacent braiding yarn with the same orientation on the composites surface and the $z$-axis and $\gamma$ is the interior braiding angle between the central axis of the interior braiding yarn and the $z$-axis. As given in Figure 3, the cross-section of the braiding yarns is the octagon containing an inscribed ellipse with major and minor radii, $a$ and $b$, respectively. The braiding yarns used in the braided preforms are assumed to have identical constituent material, size, and flexibility. The relation of geometry parameters of the above RVE model can be calculated as follows:

$$
\begin{gathered}
\tan \gamma=\sqrt{2} \tan \alpha, \\
W_{i}=T_{i}=4 \sqrt{2} b, \\
h=\frac{8 b}{\tan \gamma}, \\
a=\sqrt{3} b \cos \gamma,
\end{gathered}
$$



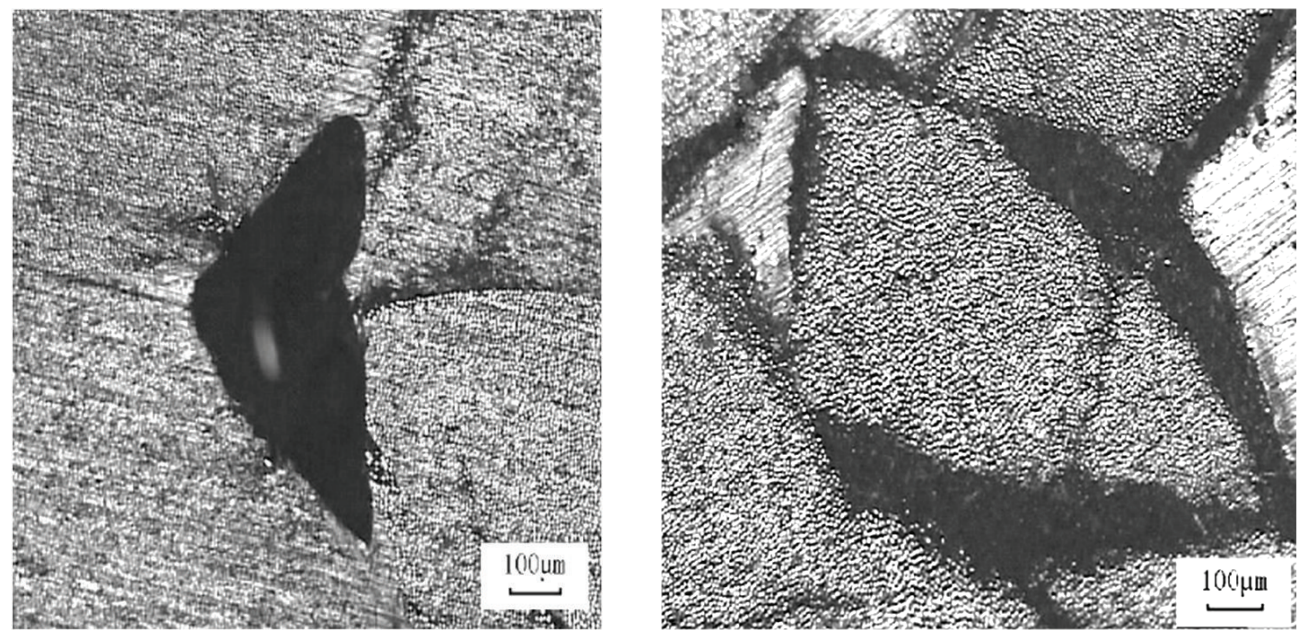

FIGURE 2: Cross-sectional shape of voids in resin matrix pocket of 3D braided composites [29].
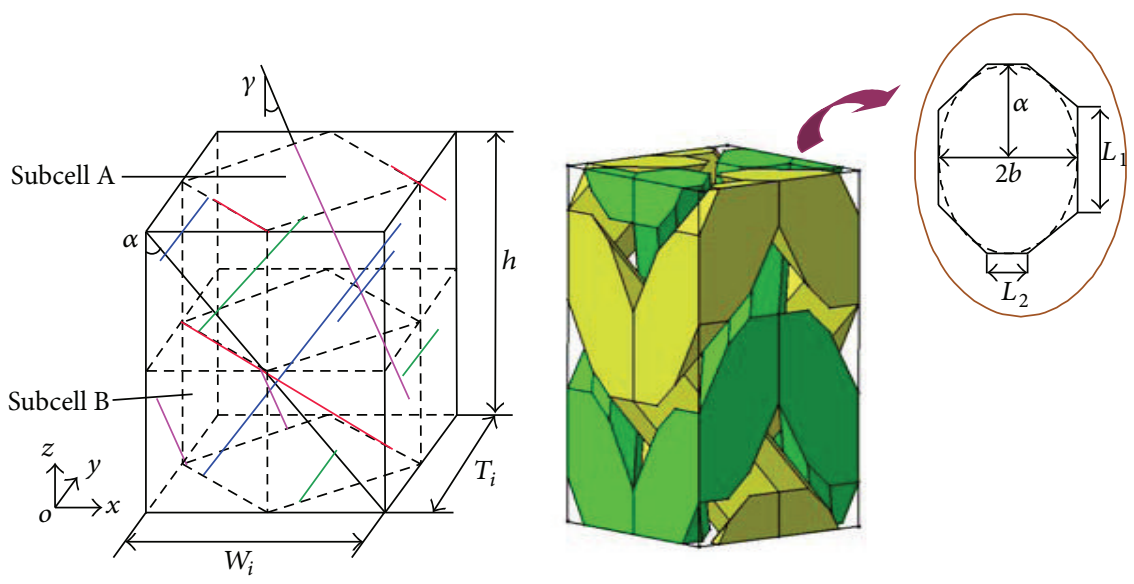

FIGURE 3: Representative unit cell model.

$$
\begin{gathered}
L_{1}=2 b \cos \gamma, \\
L_{2}=(4-2 \sqrt{3}) b, \\
S_{i}=8(\sqrt{3}-1) b^{2} \cos \gamma,
\end{gathered}
$$

where the braiding angle $\alpha$ and the pitch length $h$ of the RVE can be measured directly from the surface of the rectangular composites. As the idealized braided composites considered herein are assumed to be made of the repeated interior unit cells, the fiber volume fraction of the interior unit cell or 3D braided composites can be written as

$$
V_{f}=\frac{V_{y}}{W_{i} \times T_{i} \times h} \varepsilon,
$$

where $V_{y}=4 h S_{i} / \cos \gamma$ is the volume of all the braiding yarns in the unit cell. Since the fiber volume fraction of 3D braided composites $V_{f}$ is given usually, the fiber volume fraction of yarn $\varepsilon$ can be obtained. Once the braiding angle, the pitch length $h$, and the fiber volume fraction $V_{f}$ of $3 \mathrm{D}$ braided composites are obtained, the other geometrical parameters of the RVE unit cell model can be calculated according to formulas (3)-(3).

\section{Mesomechanical FEM Containing Void Defects}

The RVE-based mesomechanical FEM consists of three parts: establishing the FEM with random void defects, defining the constitutive relationships of components, and calculating the effective elastic properties. The details of the FEM are presented in the subsections.

4.1. FEM with Random Void Defects. Based on the interior unit cell model, a simple mesomechanical FEM containing the voids is established. In order to quantitatively analyze the effect of void defects on the elastic properties of $3 \mathrm{D}$ braided composites, it is important to effectively describe the void volume fraction, the distribution characteristics, and the geometric shape of the void defects. Considering the feature of random distribution and the complicated geometric shape 
of the void defects, the C3D4 tetrahedron elements are chosen to mesh the unit cell model because this kind of element type not only can make the meshing of unit cell model with complicated yarn microstructures relatively easy but also can well embody the main geometric characteristics of the void defects.

The void volume fraction of the composites is defined as the ratio of the void volume to the unit cell volume. In the model, $P_{y v}$ is the void volume fraction of the tows and $P_{m v}$ is the void volume fraction of the resin matrix pocket. To quantitatively appraise the effect of dry patches and voids on the elastic properties, the balanced distribution of void and dry patches is assumed in the present model. In fact, the assumption shows that the distribution characteristic of void defects keeps even as a whole in the composites due to the complicated reasons. Otherwise, it is difficult to analyze the effect of void defects on the mechanical performance if no limitation was given to the whole distribution characteristic. Based on the assumption, $P_{y v}$ and $P_{m v}$ may be believed to be equal; that is, the void volume fraction of the tows equals the void volume fraction of the resin pocket matrix as a whole. Then the void volume of the two components can be, respectively, calculated combined with the yarn volume fraction of the unit cell and the yarn packing factor. Finally, based on the averaging element volume of the tetrahedron element in the FEM, the element number of the dry patches in the yarns $N_{y}$ and the element number of void in the resin matrix pocket $N_{m}$ can be obtained by the following formula:

$$
N_{y}=\frac{V_{y} * P_{y v}}{\overline{V_{e}} *\left(1-\varepsilon_{i}\right)}, \quad N_{m}=\frac{\left(V_{u}-V_{y}\right) * P_{m v}}{\overline{V_{e}}},
$$

where $V_{u}$ is the unit cell volume, $V_{y}$ is the yarn volume fraction of the unit cell, $\overline{V_{e}}$ is the averaging element volume of the tetrahedron element, and $\varepsilon_{i}$ is the yarn packing factor. During establishing the FE model, the geometrical model is firstly meshed by Hypermesh software and then imported to ABAQUS. By Hypermesh software, the "volume tetra" method is chosen for meshing the model. In this step, the "element size" can be set as a specified value. Meanwhile, a reasonable element size should be chosen not only to meet the computational convergence of FEM but also to embody the geometric characteristics of the void defects. So, enough elements should be produced in the FEMs and it is helpful for the tetrahedron elements to have equivalent volumes by increasing the number of elements. Based on the above reasons, $\overline{V_{e}}$ represents the averaging element volume of the tetrahedron element and the element number of voids defects can be calculated approximately by formula (4).

The void defects of 3D braided composites include two basic types; one is the dry patches of yarn and the other is the voids in the resin matrix pocket. Since the distribution characteristics of the void defects are random, it is important to present a reasonable random distribution model for selecting the elements representing as the dry patches or the voids. Considering the complicated reasons for the generation of void defects, a probability model for producing the uniformly distributed random integers is chosen to determine the defects elements according to the element number herein. The probability model is given as

$$
\begin{gathered}
R_{i}=\bmod \left(5 * R_{i-1}, 4 * 2^{k}\right), \\
\mathrm{RND}_{i}=\operatorname{INT}\left(\frac{R_{i}}{4}\right),
\end{gathered}
$$

where $k=\left[\log _{2} S\right]+1, S=b-a+1, R_{0}$ is the random seed number, $R_{0} \geq 1$, and $R_{0}$ is an odd number, and $a$ and $b$ are the number range of the corresponding elements. Based on the probability model, every element in the unit cell model has the same chance becoming the defect element. To establish the FEM containing the void defects by using the above model, a small procedure was prepared by using FORTRAN language. Particularly, it is very important to determine the averaging size of tetrahedron element when the FEM is meshed. A reasonable element size should be chosen to meet the computational convergence of FEM and embody the geometric characteristics of the void defects. Based on the above probability model, it is convenient that an FEM model containing the void defects can be established by choosing a different odd number for $R_{0}$. According to the scheme, as an example, the elements containing the void defects in the resin matrix pocket and a yarn in unit cell model are shown in Figure 4, respectively.

Therefore, the generation of void defects in the model not only shows the balanced distribution characteristics of the void defects as a whole but also embodies the feature of random distribution.

4.2. Constitutive Relationships of Components. For the FEM containing the void defects, there are four "types" of component materials in the unit cell model altogether. When the component materials have no void defects, they are the yarns and the pure resin matrix pocket, respectively. In this case, the yarns are generally regarded as the unidirectional fiberreinforced composites and keep transversely isotropic in the material coordinates systems. The principal direction 1 of the material coordinates systems for a yarn is defined to be paralleled with the fiber direction. The pure resin matrix pocket is assumed to be isotropic. Both of them are believed to be linearly elastic in the model. The engineering elastic constants of the yarn can be calculated by the famous micromechanics formulae proposed by Chamis [32]:

$$
\begin{gathered}
E_{1}=\varepsilon E_{f 1}+(1-\varepsilon) E_{m}, \\
E_{2}=E_{3}=\frac{E_{m}}{1-\sqrt{\varepsilon}\left(1-E_{m} / E_{f 2}\right)}, \\
G_{12}=G_{31}=\frac{G_{m}}{1-\sqrt{\varepsilon}\left(1-G_{m} / G_{f 12}\right)}, \\
G_{23}=\frac{G_{m}}{1-\sqrt{\varepsilon}\left(1-G_{m} / G_{f 23}\right)}, \\
v_{12}=v_{13}=\varepsilon \cdot v_{f 12}+(1-\varepsilon) v_{m}, \\
v_{23}=\frac{E_{2}}{2 G_{23}}-1,
\end{gathered}
$$




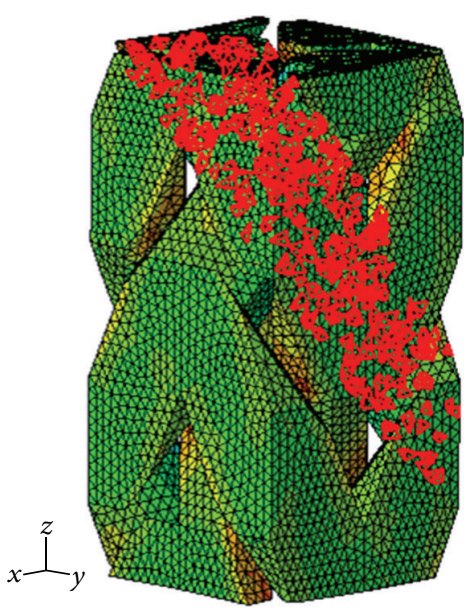

(a) Void defects in one yarn

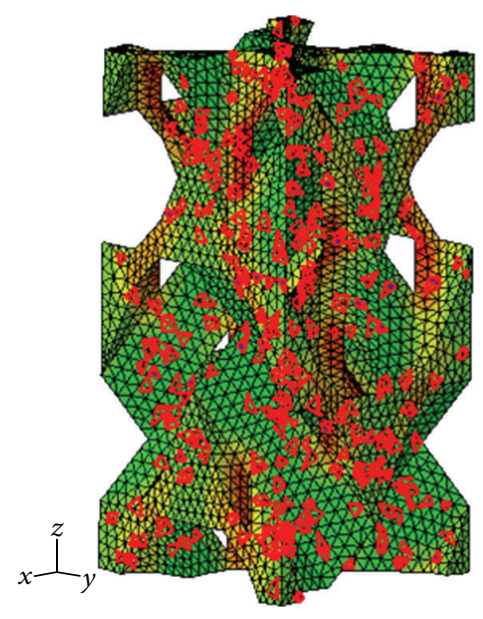

(b) Void defects in resin matrix pocket

FIGURE 4: Random distribution of void defects in FEM.

where $\varepsilon$ is the fiber volume fraction of the yarn, $E_{f 1}$ is Young's elastic modulus of the fiber in principle axis $1, E_{f 2}$ is Young's elastic modulus of the fiber in principle axis $2, G_{f 12}$ is the longitudinal shear modulus of the fiber, $G_{f 23}$ is the transverse shear modulus of the fiber, $v_{f 12}$ is the primary Poisson's ratio of the fiber, and $E_{m}, v_{m}$, and $G_{m}\left(G_{m}=E_{m} / 2\left(1+v_{m}\right)\right)$ represent Young's elastic modulus, Poisson's ratio, and shear modulus of the matrix, respectively.

When the component materials contain the void defects, the elements of the component materials can be classified as two groups. They are the elements of the dry patches of yarn and the void elements in the pure resin matrix pocket, respectively. For the elements of the dry patches of yarn, there are only fibers in the elements of yarns without resin infiltration. As we know, the main role of resin matrix in yarns is to support and protect the fibers, which is important for the mechanical properties in the transverse and longitudinaltransverse shear directions. Therefore, it is difficult to exactly characterize the elastic properties of the dry patches of unidirectional composites where the resin matrix is replaced by the voids. To simply model the constitutive relationship of the dry patches elements, the micromechanics formulae proposed by Chamis are still chosen to calculate the effective elastic properties by assuming that Young's elastic modulus $E_{m}$ and Poisson's ratio $v_{m}$ of the resin material in such elements are reduced to be a minimum value. In the model, in order to stress that the dry patches of unidirectional composites have a certain load-bearing capability in the transverse directions, the comparably larger values are defined for the resin matrix of the dry patch element in yarns. The value of Young's elastic modulus $E_{m}$ of resin is assumed to be $1 \mathrm{MPa}$ and the value of Poisson's ratio $v_{m}$ of resin is assumed to be 0.01 . It is noted that the dry patch elements in yarns still keep the same local coordinate systems as the elements of yarns without void defects.

For the void elements in the pure resin matrix pocket, a "zero" stiffness element is chosen to simulate the constitutive relationship. To satisfy the numerical calculation demand of the FEM, a minimum value for Young's elastic modulus $E_{m}$ is designated as $0.000001 \mathrm{MPa}$ and Poisson's ratio $v_{m}$ is designated as 0.000001 , respectively.

Once the random defect elements of unit cell model are determined, the above four "types" of constitutive relationships can be applied to the corresponding element sets in the finite elements model. To achieve the goal, the INP document of FEM should be modified by revising the element section properties and the name of the corresponding element sets before the model is submitted to be calculated based on the software ABAQUS.

4.3. Effective Elastic Properties of $3 D$ Braided Composites. When the void volume fraction of 3D braided composites is given, for a specific random distribution of the void defects, 3D braided composites are assumed to be composed of the interior unit cells containing the same distribution feature of void defects. That is, the effective elastic properties of 3D braided composites are equivalent to the calculated results based on the mesoscale FE model by a homogenization approach.

Since the analysis is based on the RVE, the periodical boundary conditions should be applied in the model in order to obtain a reasonable stress distribution and deformation. The unified periodical displacement boundary conditions suitable for the RVE proposed by Xia et al. [33] were employed in the model. The 3D solid tetrahedron elements were applied to mesh the whole model, as shown in Figure 4. It is assumed that the perfect bonding exists between the yarns and the resin matrix pocket.

To obtain the effective elastic properties of 3D 4-directional braided composites, the homogenization approach is employed in this study by considering the heterogeneous composites in the mesoscale to be a homogeneous material in the macro-scale. In the mesoscale, when the void volume fraction of unit cell is given, for a specific random distribution of the void defects, the interior unit cell containing the same 
TABLE 1: Materials properties of components (modulus/GPa).

\begin{tabular}{lcccccc}
\hline & $E_{f 1}$ & $E_{f 2}$ & $G_{f 12}$ & $G_{f 23}$ & $\mu_{f 12}$ & $\mu_{m}$ \\
\hline Carbon fiber & 230 & 40 & 24 & 14.3 & 0.25 & \\
Epoxy & 3.5 & & & & & 0.35 \\
\hline
\end{tabular}

distribution feature of void defects is taken out to establish the FEM for appraising the elastic properties of 3D braided composites based on the periodic boundary conditions. Given the periodic cubic RVE, the global strain-global stress relation under small deformation assumption can be written as

$$
\bar{\varepsilon}_{i}=\mathbf{S}_{i j} \bar{\sigma}_{j}
$$

where $\mathbf{S}_{i j}$ is the effective compliance matrix, $\bar{\varepsilon}_{i j}$ is the global averaging strain, and $\bar{\sigma}_{i j}$ is the global averaging stress. The engineering elastic constants can be calculated from the compliance matrix $\mathbf{S}_{i j}$.

In the macro-scale, when the void volume fraction of $3 \mathrm{D}$ braided composites is given, there are possibly numerous random distribution cases for the void defects. To analyze the effect of random distribution of void defects on the effective elastic properties, the samples of FE model containing different defect distribution cases should be established for statistical analysis of the elastic properties of 3D braided composites with a given void volume fraction.

\section{Results and Discussion}

5.1. Comparison of Effective Elastic Properties with Experimental Results. In order to verify the applicability of the FEM based on the software ABAQUS, two examples with typical braiding angles are selected from the available experiments studied by Chen et al. [21]. All the analyses reported herein were done for the 3D 4-directional braided composites by the 4 -step $1 \times 1$ rectangular braiding procedures. The elastic properties of the component materials, including $12 \mathrm{~K}$ T300 carbon fiber and TDE-85 epoxy resin, are listed in Table 1. According to the braiding process parameters of three specimens from Chen et al. [21], the main microstructure parameters of unit cell models used in the calculation are shown in Table 2.

The FE software ABAQUS is adopted to study the effective elastic properties and the mesoscale mechanical behaviors. According to the meshing scheme of the FEM, adaptive finite element meshes were used to keep element size small in the edges of the matrix pocket. In the study, the FEM for specimen number 1 with a braiding angle of $19^{\circ}$ consists of 9854 nodes and 49030 tetrahedron elements. The FEM for specimen number 2 with a braiding angle of $30^{\circ}$ consists of 17462 nodes and 88700 tetrahedron elements. It is noted that relatively reasonable meshing size is required in order to embody the real geometric shape of void defects. If only the global stiffness is concerned, relative coarse meshing size can still provide satisfactory results [33]. The meshing size of the models in this study is sufficient to guarantee the convergence of the solutions. According to the definition of engineering elastic constants, the FEM based on the unit cells under uniaxial tensile loading along the $x$-, $y$-, and $z$-axes was established to obtain Young's elastic modulus and Poisson's ratio, respectively. In the meantime, the pure shear loading was applied to the corresponding models to analyze the shear deformation and modulus. In the calculation models, the global averaging tensile strain $\bar{\varepsilon}_{i}$ equals 0.001 and the global averaging shear strain $\bar{\varepsilon}_{i j}$ equals 0.002 .

Table 3 gives the predicted elastic constants of 3D braided composite without considering the void defects and the measured results. There is a good agreement between the predicted and measured axial tensile modulus for the two samples studied. Predicted Poisson's ratios basically agreed with the measured values. The results indicate that the proposed FEM can be used to calculate the effective elastic properties, firstly demonstrating the applicability of the mesomechanical FEM.

\subsection{Stress Distribution and Deformation of Unit Cell Model.} For 3D 4-directional braided composites with periodic structures, RVE-based FEM containing void defects can be used to calculate the mechanical properties in the mesoscale, such as the deformation of the model, distribution of stress, and stress concentration. To demonstrate the application, the FEM of specimen number 1 subjected to typical loading cases is chosen to show the mesomechanical behaviors of 3D 4directional braided composites by assuming that the void volume fraction equals 1 percent.

By analyzing the numerical results of the model, all the stress components at the corresponding parallel boundary surfaces have a uniform stress distribution. For example, Figure 5 shows the Von Mises stress nephogram of the whole FEM subjected to $z$ tension and $x z$ shear loads, respectively. The traction continuity at the corresponding parallel boundary surfaces has been guaranteed and satisfied the periodic condition. As shown in Figure 5, the stress in yarns is apparently greater than that in the matrix pocket region. In the meantime, the stress concentration is produced in the contacting region between the yarns and the matrix pocket. The closer it is to this region, the greater stress is produced.

Figure 6 shows the deformation of the unit cell models subjected to $z$ tension and $x z$ shear loads, respectively. From Figure 6(a), the set of opposite boundary surfaces vertical to the $z$-axis, respectively, do not remain plane after deformation and the warped deformation has occurred (the magnified factor of the deformation is 100 times). The same warped deformation occurs simultaneously at the opposite boundary surfaces vertical to the $z$-axis. However, the warped deformation extent at the other two sets of opposite boundary surfaces vertical to the $x$-axis and $y$-axis is relatively weak. Figure 6(b) shows the deformation of the FEM model subjected to $x z$ shear load. Under such loading case, two sets of opposite boundary surfaces in the $x z$ plane do not remain plane anymore and are warped after the deformation (the magnified factor of the deformation is 100 times). The warped deformation occurs simultaneously at the other set of opposite boundary surfaces of unit cell vertical to $y$-axis, but the warped extent is relatively weak. The reason that 
TABLE 2: Braiding parameters of specimens and structural parameters of unit cell model.

\begin{tabular}{|c|c|c|c|c|c|c|c|c|c|}
\hline Number & Dimensions $(\mathrm{mm})$ & $\alpha\left(^{\circ}\right)$ & $V_{f}(\%)$ & $\gamma\left({ }^{\circ}\right)$ & $a(\mathrm{~mm})$ & $b(\mathrm{~mm})$ & $W_{i}=T_{i}(\mathrm{~mm})$ & $h(\mathrm{~mm})$ & $\varepsilon(\%)$ \\
\hline Number 1 & $20 \times 6 \times 250$ & 19 & 46.6 & 26.0 & 0.599 & 0.385 & 2.175 & 6.317 & 63.66 \\
\hline Number 2 & $20 \times 6 \times 250$ & 30 & 47.2 & 39.2 & 0.542 & 0.404 & 2.286 & 3.960 & 64.48 \\
\hline
\end{tabular}

TABLE 3: Comparison of the elastic constants predicted by the model and experimental data.

\begin{tabular}{|c|c|c|c|c|}
\hline \multirow{2}{*}{ Elastic constants } & \multicolumn{2}{|c|}{ Number 1} & \multicolumn{2}{|c|}{ Number 2} \\
\hline & Experiment & Predicted & Experiment & Predicted \\
\hline$E_{x} / \mathrm{GPa}$ & & 9.01 & & 9.04 \\
\hline$E_{y} / \mathrm{GPa}$ & & 9.01 & & 9.04 \\
\hline$E_{z} / \mathrm{GPa}$ & 58.74 & 60.32 & 27.60 & 25.66 \\
\hline$G_{x z} / \mathrm{GPa}$ & & 11.41 & & 15.70 \\
\hline$G_{y z} / \mathrm{GPa}$ & & 11.41 & & 15.70 \\
\hline$G_{x y} / \mathrm{GPa}$ & & 4.31 & & 7.50 \\
\hline$\mu_{x z}$ & 0.72 & 0.69 & 0.78 & 0.69 \\
\hline$\mu_{y z}$ & 0.69 & 0.69 & 1.00 & 0.69 \\
\hline$\mu_{x y}$ & & 0.35 & & 0.30 \\
\hline
\end{tabular}

resulted in the phenomena is that the unit cell model of 3D 4directional braided composites does not have the symmetries of geometrical structure and physical properties.

\subsection{Effect of Random Void Distribution on the Effective Elastic} Properties. When the void volume fraction is given, the void defects may be randomly distributed in the unit cell model of 3D braided composites due to the complex reasons. Therefore, it is important to analyze the effect of random distribution of void defects on the effective elastic properties of 3D braided composites. Herein, specimen number 1 in Table 2 was taken as an example for analyzing the effect of random void distribution on the elastic properties by assuming that the void volume fraction equals 1 percent. For simplicity, five random void distribution models named $R_{1}$ to $R_{5}$ for specimen number 1 were produced to predict the elastic properties subjected to the uniaxial tensile loading in $z$-axis and the shear loading in $x z$ plane, respectively. The calculated results are shown in Table 4, where the elastic moduli are corrected to 3 decimal places and Poisson's ratios are corrected to 4 decimal places.

As shown in Table 4, when the void volume fraction is given as $1 \%$, the standard deviation of the tensile modulus $E_{z}$ is no more than $3.138 E-3 \mathrm{GPa}$ and the standard deviation of the shear modulus $G_{x z}$ is limited to $4.967 E-4 \mathrm{GPa}$. The standard deviations of Poisson's ratios, $\mu_{x z}$ and $\mu_{y z}$, are within $1.0198 E-4$ and $2.2804 E-4$, respectively. From the results, it can be concluded that the elastic properties are not sensitive to the random void distribution for $3 \mathrm{D}$ braided composites with a constant void volume fraction. The resulting reason could be that the stiffness properties reflect the global averaging properties of 3D braided composites, which mainly depends on the mechanical properties and their volume fractions of components materials. However, it is noted that all the results are based on the premise of the distribution of random void defects keeping balanced in the model as a whole without considering some extremely special distribution cases.

5.4. Effect of Void Volume Fraction on the Effective Elastic Properties. As discussed above, the random distribution of void defects has no obvious effect on the effective elastic properties of $3 \mathrm{D}$ braided composites in the model. Since the most important impact factor is the void volume fraction of $3 \mathrm{D}$ braided composites, the effects of the void volume fraction ranging from zero to three percent on the elastic properties are discussed by analyzing the FEMs. Variations of the calculated elastic properties with the void volume fraction are shown in Tables 5 and 6 , respectively.

Table 5 gives the calculated results of specimen number 1 and Table 6 gives the calculated results of specimen number 2 . From Tables 5 and 6 , when the void volume fraction increases from zero to three percent, whether specimen number 1 with a braiding angle of $19^{\circ}$ or specimen number 2 with a braiding angle of $30^{\circ}$, all the elastic moduli including Young's elastic modulus and the shear modulus monotonously decrease and the decline trends almost keep linear. It is noted that the decline extent of transverse Young's elastic moduli, $E_{x}$ and $E_{y}$, is obviously greater than the axial modulus $E_{z}$. Specifically, when the void volume fraction reaches three percent, the Young's elastic modulus $E_{z}$ of specimen number 1 has declined by 1.45 percent, while Young's elastic modulus $E_{z}$ of specimen number 2 has declined up to 4.28 percent. Due to the two specimens almost having the same fiber volume fraction, the effect of the void volume fraction on $E_{z}$ becomes more obvious with the increase of the braiding angle. In the meantime, transverse Young's elastic moduli, $E_{x}$ and $E_{y}$, have decreased by 7.31 percent and 7.56 percent, respectively. When the void volume fraction reaches 3 percent, the shear moduli of specimen number $1, G_{x z}$ and $G_{y z}$, decrease about 2.04 percent and the shear moduli of specimen number 2, $G_{x z}$ and $G_{y z}$, decrease about 1.27 percent. In the meantime, 


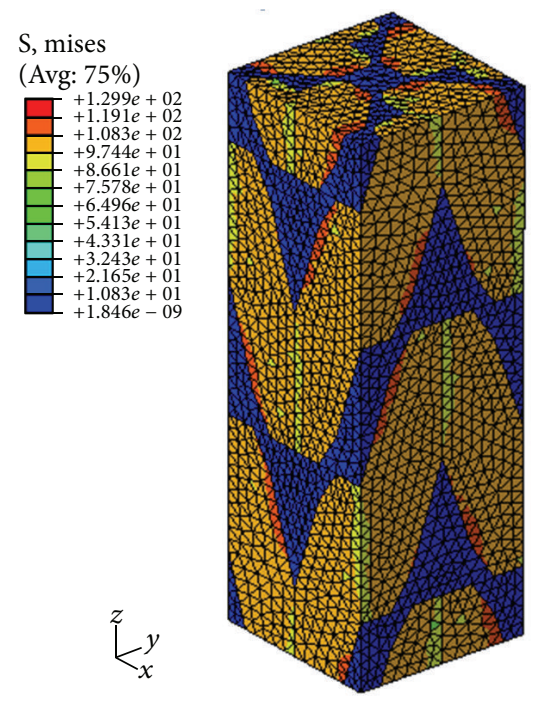

(a) Stress nephogram of the unit cell under $z$ tension load

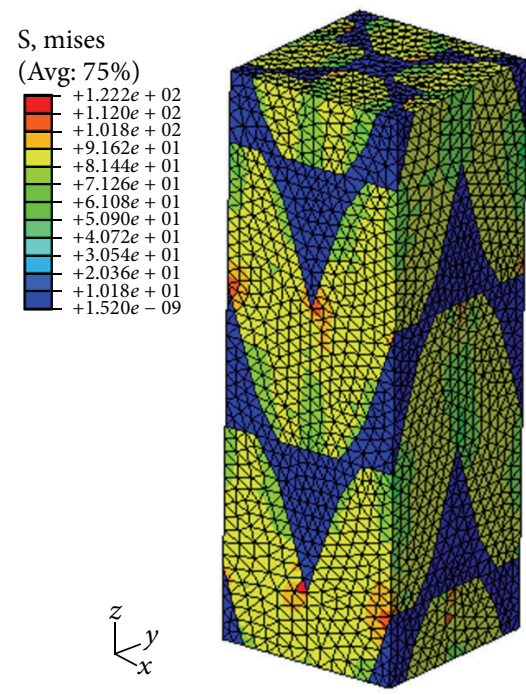

(b) Stress nephogram of the unit cell under $x z$ shear load

FIGURE 5: Stress nephogram of the FEM.

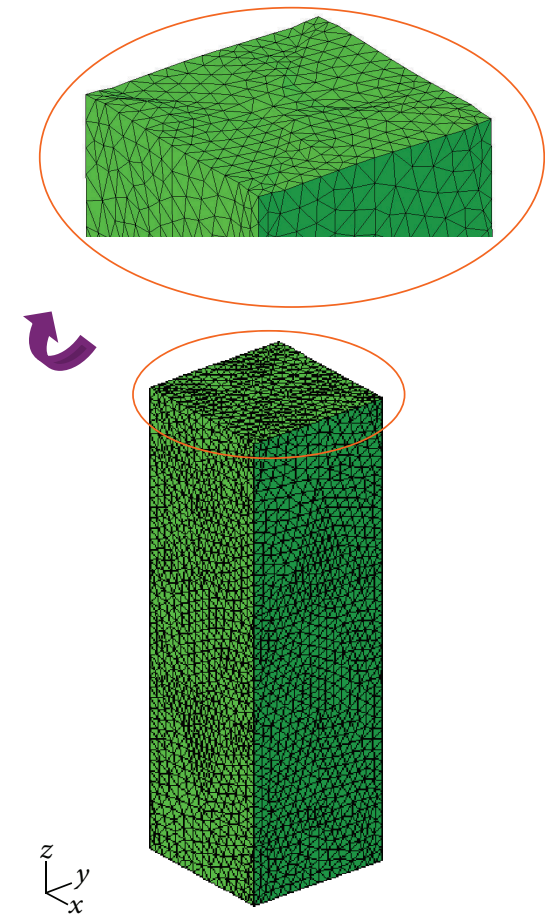

(a) Deformation of the unit cell under $z$ tension load

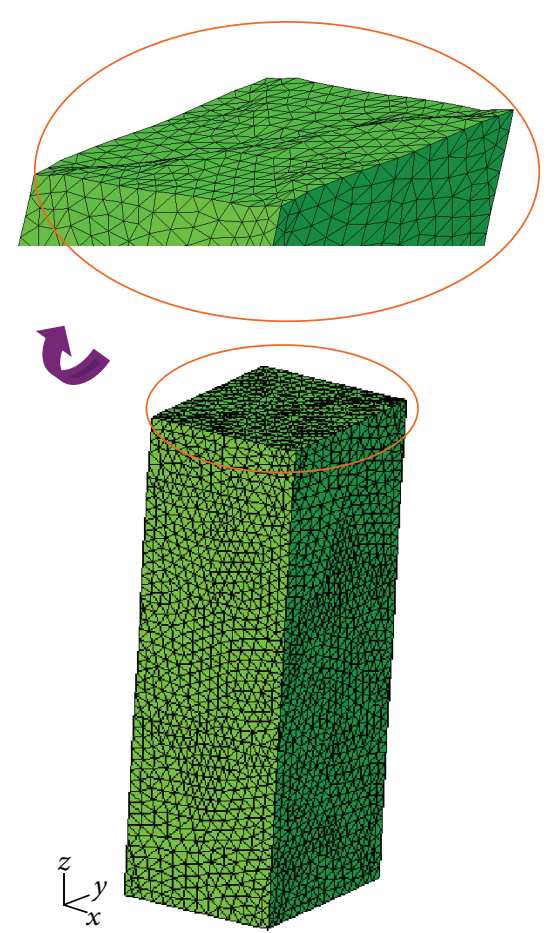

(b) Deformation of the unit cell under $x z$ shear load

FIgURE 6: Deformation of the FEM.

TABLE 4: Effect of the random distribution of the void defects on the elastic properties.

\begin{tabular}{lcccccccc}
\hline Elastic constants & $R_{1}$ & $R_{2}$ & $R_{3}$ & $R_{4}$ & $R_{5}$ & Mean value $(k)$ & Standard deviation $(s)$ & $s / k$ \\
\hline$E_{z} / \mathrm{GPa}$ & 60.073 & 60.082 & 60.082 & 60.078 & 60.081 & 60.079 & $3.138 E-3$ & $5.222 E-05$ \\
$\mu_{x z}$ & 0.7029 & 0.7031 & 0.7032 & 0.7030 & 0.7030 & 0.7030 & $1.0198 E-4$ & $1.4506 E-04$ \\
$\mu_{y z}$ & 0.7033 & 0.7028 & 0.7026 & 0.7029 & 0.7029 & 0.7029 & $2.2804 E-4$ & $3.2442 E-04$ \\
$G_{x z} / \mathrm{GPa}$ & 11.345 & 11.346 & 11.345 & 11.345 & 11.346 & 11.345 & $4.967 E-4$ & $4.378 E-05$ \\
\hline
\end{tabular}


TABLE 5: Effect of $p_{y v}$ (or $p_{m v}$ ) on the elastic properties of specimen number 1 .

\begin{tabular}{|c|c|c|c|c|c|c|c|}
\hline $\begin{array}{l}\text { Elastic } \\
\text { constants }\end{array}$ & $p_{y v}=p_{m v}=0 \%$ & $p_{y v}=p_{m v}=0.5 \%$ & $p_{y v}=p_{m v}=1.0 \%$ & $p_{y v}=p_{m v}=1.5 \%$ & $p_{y v}=p_{m v}=2.0 \%$ & $p_{y v}=p_{m v}=2.5 \%$ & $p_{y v}=p_{m v}=3.0 \%$ \\
\hline$E_{x} / \mathrm{GPa}$ & 9.011 & 8.912 & 8.800 & 8.690 & 8.580 & 8.477 & 8.361 \\
\hline$E_{y} / \mathrm{GPa}$ & 9.011 & 8.912 & 8.802 & 8.694 & 8.585 & 8.472 & 8.361 \\
\hline$E_{z} / \mathrm{GPa}$ & 60.322 & 60.225 & 60.079 & 59.935 & 59.787 & 59.644 & 59.488 \\
\hline$G_{x z} / \mathrm{GPa}$ & 11.413 & 11.385 & 11.346 & 11.306 & 11.267 & 11.232 & 11.191 \\
\hline$G_{y z} / \mathrm{GPa}$ & 11.413 & 11.386 & 11.347 & 11.309 & 11.271 & 11.230 & 11.191 \\
\hline$G_{x y} / \mathrm{GPa}$ & 4.314 & 4.285 & 4.244 & 4.203 & 4.160 & 4.121 & 4.080 \\
\hline$\mu_{x z}$ & 0.6922 & 0.6979 & 0.7030 & 0.7083 & 0.7137 & 0.7184 & 0.7246 \\
\hline$\mu_{y z}$ & 0.6923 & 0.6979 & 0.7029 & 0.7080 & 0.7133 & 0.7189 & 0.7244 \\
\hline$\mu_{x y}$ & 0.3511 & 0.3474 & 0.3454 & 0.3473 & 0.3472 & 0.3472 & 0.3470 \\
\hline
\end{tabular}

TABLE 6: Effect of $p_{y v}\left(\right.$ or $p_{m v}$ ) on the elastic properties of specimen number 2.

\begin{tabular}{|c|c|c|c|c|c|c|c|}
\hline $\begin{array}{l}\text { Elastic } \\
\text { constants }\end{array}$ & $p_{y v}=p_{m v}=0 \%$ & $p_{y v}=p_{m v}=0.5 \%$ & $p_{y v}=p_{m v}=1.0 \%$ & $p_{y v}=p_{m v}=1.5 \%$ & $p_{y v}=p_{m v}=2.0 \%$ & $p_{y v}=p_{m v}=2.5 \%$ & $p_{y v}=p_{m v}=3.0 \%$ \\
\hline$E_{x} / \mathrm{GPa}$ & 9.042 & 8.929 & 8.813 & 8.703 & 8.588 & 8.476 & 8.358 \\
\hline$E_{y} / \mathrm{GPa}$ & 9.042 & 8.932 & 8.821 & 8.702 & 8.589 & 8.478 & 8.363 \\
\hline$E_{z} / \mathrm{GPa}$ & 25.661 & 25.488 & 25.304 & 25.123 & 24.940 & 24.754 & 24.568 \\
\hline$G_{x z} / \mathrm{GPa}$ & 15.701 & 15.662 & 15.629 & 15.596 & 15.562 & 15.529 & 15.495 \\
\hline$G_{y z} / \mathrm{GPa}$ & 15.701 & 15.663 & 15.630 & 15.596 & 15.563 & 15.529 & 15.496 \\
\hline$G_{x y} / \mathrm{GPa}$ & 7.503 & 7.464 & 7.425 & 7.385 & 7.346 & 7.306 & 7.267 \\
\hline$\mu_{x z}$ & 0.6912 & 0.6890 & 0.6932 & 0.6965 & 0.7005 & 0.7045 & 0.7087 \\
\hline$\mu_{y z}$ & 0.6913 & 0.6888 & 0.6922 & 0.6966 & 0.7004 & 0.7041 & 0.7080 \\
\hline$\mu_{x y}$ & 0.3022 & 0.2992 & 0.2992 & 0.2990 & 0.2990 & 0.2989 & 0.2990 \\
\hline
\end{tabular}

the shear modulus of specimen number $1 G_{x y}$ decreases about 5.69 percent and the shear modulus of specimen number 2 $G_{x y}$ decreases about 3.14 percent. On the whole, the effect of the void volume fraction on shear modulus becomes weak with the increase of the braiding angle.

From Tables 5 and 6, when the void volume fraction increases from zero to three percent, whether specimen number 1 with a braiding angle of $19^{\circ}$ or specimen number 2 with a braiding angle of $30^{\circ}$, primary Poisson's ratios, $\mu_{x z}$ and $\mu_{y z}$, increase monotonously and the increase trends almost keep linear, while minor Poisson's ratio $\mu_{x y}$ decreases steadily. Specifically, while the void volume fraction reaches 3 percent, primary Poisson's ratios of specimen number $1, \mu_{x z}$ and $\mu_{y z}$, increase up to about 4.54 percent and primary Poisson's ratios of specimen number $2, \mu_{x z}$ and $\mu_{y z}$, increase about 3.37 percent. In the meantime, particularly, minor Poisson's ratios of specimen number 1 and specimen number $2, \mu_{x y}$, decline no more than 0.15 percent when the void volume fraction equals 3 percent. As a whole, the void volume fraction mainly has great effect on primary Poisson's ratio, $\mu_{x z}$ and $\mu_{y z}$, while it almost has little effect on the minor Poisson's ratio $\mu_{x y}$.

\section{Conclusions}

An FEM based on the RVE is proposed to predict the effective elastic properties of 3D braided composites with consideration of random void defects. The 3D FEM model based on the periodical unit cells takes into account the voids in the resin matrix pocket and the dry patches in the yarns. The predicted effective elastic properties are compared favorably with the experimental data, demonstrating the applicability of the mesomechanical FEM. The effects of the void random distribution and the void volume fraction on the engineering elastic constants have been discussed in detail. The calculated results show that the elastic properties of $3 \mathrm{D}$ braided composites are influenced significantly by the void volume fraction. While all the elastic moduli decrease almost linearly with the increase of the void volume fraction, $\mu_{x z}$ and $\mu_{y z}$ increase almost linearly except for $\mu_{x y}$. The effect of the void volume fraction on the elastic constant $E_{z}$ becomes more obvious with increase of the braiding angle.

Future work will focus on the effect of void defects on the strength, thermal conductivity, and thermal expansion properties of the composites in the subsequent research. 


\section{Conflict of Interests}

The authors declare that there is no conflict of interests regarding the publication of this paper.

\section{Acknowledgments}

The authors would like to acknowledge the support provided by the Fundamental Research Funds for the Central Universities (Grant no. ZYGX2011J122) and the National Natural Science Foundation of China (Grant no. 11302045).

\section{References}

[1] K. Woo and J. Whitcomb, "Global/local finite element analysis for textile composites," Journal of Composite Materials, vol. 28, no. 14, pp. 1305-1321, 1994.

[2] J. Whitcomb and K. Srirengan, "Effect of various approximations on predicted progressive failure in plain weave composites," Composite Structures, vol. 34, no. 1, pp. 13-20, 1996.

[3] J. D. Whitcomb, C. D. Chapman, and K. Srirengan, "Analysis of plain-weave composites subjected to flexure," Mechanics of Composite Materials and Structures, vol. 5, no. 1, pp. 41-53, 1998.

[4] X. D. Tang and J. D. Whitcomb, "General techniques for exploiting periodicity and symmetries in micromechanics analysis of textile composites," Journal of Composite Materials, vol. 37, no. 13, pp. 1167-1189, 2003.

[5] X. D. Tang, J. D. Whitcomb, A. D. Kelkar, and J. Tate, "Progressive failure analysis of $2 \times 2$ braided composites exhibiting multiscale heterogeneity," Composites Science and Technology, vol. 66, no. 14, pp. 2580-2590, 2006.

[6] S. V. Lomov, A. V. Truevtzev, and C. Cassidy, "A predictive model for the fabric-to-yarn bending stiffness ratio of a plainwoven set fabric," Textile Research Journal, vol. 70, no. 12, pp. 1088-1096, 2000.

[7] I. Verpoest and S. V. Lomov, "Virtual textile composites software WiseTex: integration with micro-mechanical, permeability and structural analysis," Composites Science and Technology, vol. 65, no. 15-16, pp. 2563-2574, 2005.

[8] Q. D. Yang, K. L. Rugg, B. N. Cox, and M. C. Shaw, "Failure in the junction region of T-stiffeners: 3D-braided vs. 2D tape laminate stiffeners," International Journal of Solids and Structures, vol. 40, no. 7, pp. 1653-1668, 2003.

[9] Q. D. Yang and B. N. Cox, "Spatially averaged local strains in textile composites via the binary model formulation," Journal of Engineering Materials and Technology, vol. 125, no. 4, pp. 418425, 2003.

[10] Q. D. Yang, K. L. Rugg, B. N. Cox, and D. B. Marshall, "Evaluation of macroscopic and local strains in a three-dimensional woven $\mathrm{C} / \mathrm{SiC}$ composite," Journal of the American Ceramic Society, vol. 88, no. 3, pp. 719-725, 2005.

[11] Q. D. Yang and B. N. Cox, "Predicting failure in textile composites using the Binary Model with gauge-averaging," Engineering Fracture Mechanics, vol. 77, no. 16, pp. 3174-3189, 2010.

[12] B. N. Cox and Q. D. Yang, "In quest of virtual tests for structural composites," Science, vol. 314, no. 5802, pp. 1102-1107, 2006.

[13] R. L. Karkkainen and B. V. Sankar, "A direct micromechanics method for analysis of failure initiation of plain weave textile composites," Composites Science and Technology, vol. 66, no. 1, pp. 137-150, 2006.
[14] R. L. Karkkainen, B. V. Sankar, and J. T. Tzeng, "Strength prediction of multi-layer plain weave textile composites using the direct micromechanics method," Composites B, vol. 38, no. 7-8, pp. 924-932, 2007.

[15] R. L. Karkkainen and J. T. Tzeng, "Micromechanical strength modeling and investigation of stitch density effects on 3D orthogonal composites," Journal of Composite Materials, vol. 43, no. 25, pp. 3125-3142, 2009.

[16] S. C. Quek, A. Waas, K. W. Shahwan, and V. Agaram, "Compressive response and failure of braided textile composites: part 2computations," International Journal of Non-Linear Mechanics, vol. 39, no. 4, pp. 649-663, 2004.

[17] E. J. Pineda, A. M. Waas, B. A. Bednarcyk, C. S. Collier, and P. W. Yarrington, "Progressive damage and failure modeling in notched laminated fiber reinforced composites," International Journal of Fracture, vol. 158, no. 2, pp. 125-143, 2009.

[18] J.-M. Yang, C.-L. Ma, and T.-W. Chou, "Fiber inclination model of three dimensional textile structural composites," Journal of Composite Materials, vol. 20, no. 5, pp. 472-484, 1986.

[19] Y.-Q. Wang and A. S. D. Wang, "Microstructure/property relationships in three-dimensionally braided fiber composites," Composites Science and Technology, vol. 53, no. 2, pp. 213-222, 1995.

[20] X. K. Sun and C. J. Sun, "Mechanical properties of three-dimensional braided composites," Composite Structures, vol. 65, no. 34, pp. 485-492, 2004.

[21] L. Chen, X. M. Tao, and C. L. Choy, "Mechanical analysis of 3-D braided composites by the finite multiphase element method," Composites Science and Technology, vol. 59, no. 16, pp. 23832391, 1999.

[22] K. Xu and X. W. Xu, "Finite element analysis of mechanical properties of 3D five-directional braided composites," Materials Science and Engineering A, vol. 487, no. 1-2, pp. 499-509, 2008.

[23] Z. X. Lu, B. Xia, and Z. Y. Yang, "Investigation on the tensile properties of three-dimensional full five-directional braided composites," Computational Materials Science, vol. 77, pp. 445455, 2013.

[24] J. Liang, S. Y. Du, and J. C. Han, "Effective elastic properties of three dimensional braided composites with matrix microcracks," Acta Materiae Compositae Sinica, vol. 14, no. 1, pp. 101107, 1997.

[25] T. Zeng, D. N. Fang, L. C. Guo, and L. Ma, "A mechanical model of $3 \mathrm{D}$ braided composites with transverse and longitudinal cracks," Composite Structures, vol. 69, no. 1, pp. 117-125, 2005.

[26] Z. X. Lu, Q. Xu, B. P. Wang, and Z. Yang, "Numerical simulation of plain weave composites with defects under unidirectional tension," Acta Materiae Compositae Sinica, vol. 28, no. 6, pp. 200-207, 2011.

[27] H. Bale, M. Blacklock, M. R. Begley, D. B. Marshall, B. N. Cox, and R. O. Ritchie, "Characterizing three-dimensional textile ceramic composites using synchrotron X-ray micro-computedtomography," Journal of the American Ceramic Society, vol. 95, no. 1, pp. 392-402, 2012.

[28] M. Blacklock, H. Bale, M. Begley, and B. Cox, "Generating virtual textile composite specimens using statistical data from micro-computed tomography: 1D tow representations for the Binary Model," Journal of the Mechanics and Physics of Solids, vol. 60, no. 3, pp. 451-470, 2012.

[29] X.-Q. Wu and Q.-W. Guo, "Void morphology in 3-D braided preform/phenolic composites," Journal of Donghua University, vol. 26, no. 5, pp. 539-543, 2009. 
[30] Y. K. Hamidi, L. Aktas, and M. C. Altan, "Formation of microscopic voids in resin transfer molded composites," Journal of Engineering Materials and Technology, vol. 126, no. 4, pp. 420426, 2004.

[31] N. Kuentzer, P. Simacek, S. G. Advani, and S. Walsh, "Correlation of void distribution to VARTM manufacturing techniques," Composites A, vol. 38, no. 3, pp. 802-813, 2007.

[32] C. C. Chamis, "Mechanics of composite materials: past, present, and future," Journal of Composites, Technology and Research, vol. 11, no. 1, pp. 3-14, 1989.

[33] Z. H. Xia, C. W. Zhou, Q. L. Yong, and X. Wang, "On selection of repeated unit cell model and application of unified periodic boundary conditions in micro-mechanical analysis of composites," International Journal of Solids and Structures, vol. 43, no. 2, pp. 266-278, 2006. 

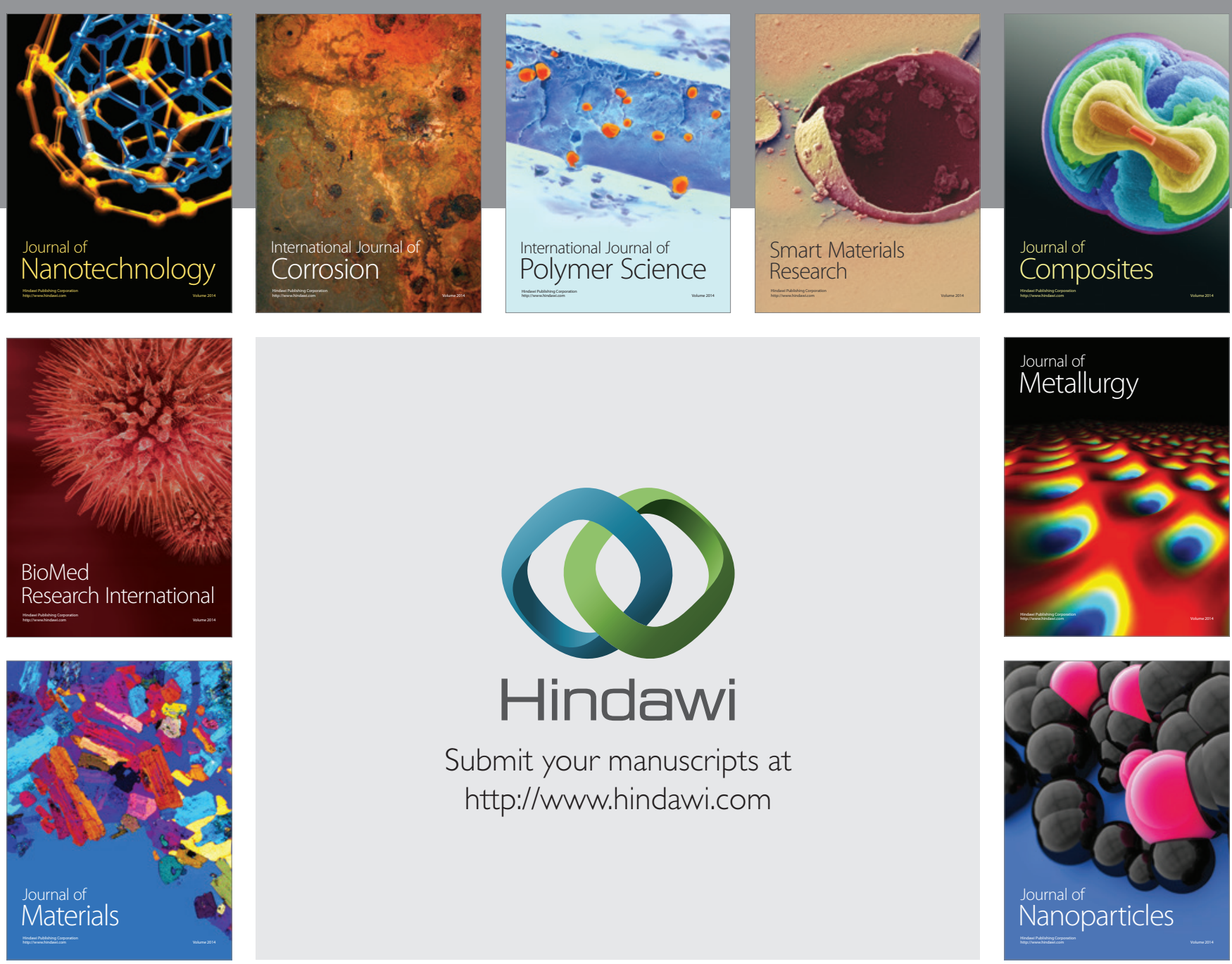

Submit your manuscripts at http://www.hindawi.com
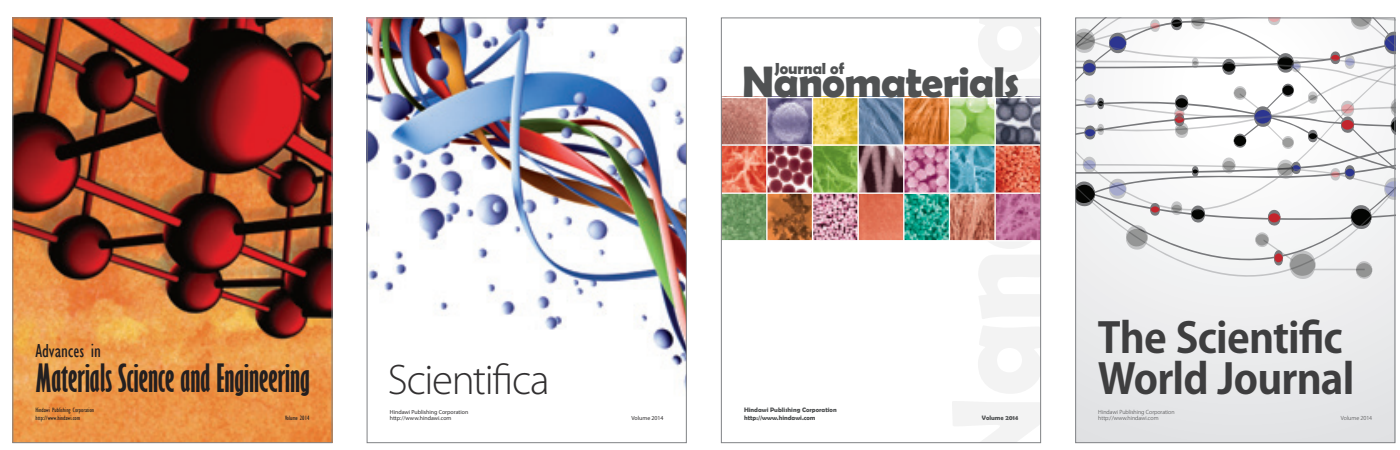

\section{The Scientific World Journal}
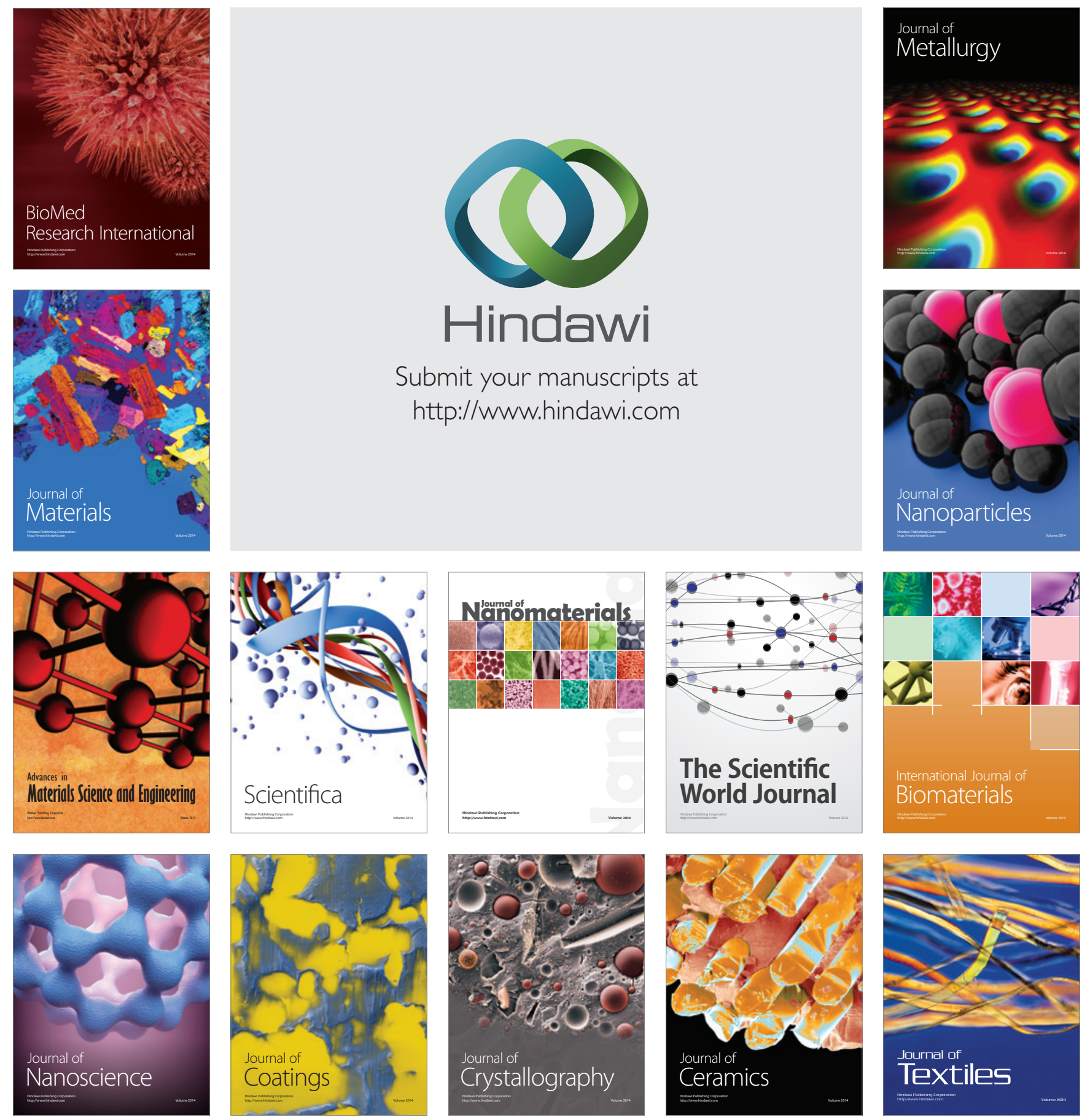